\title{
A novel contour extraction algorithm based on dynamic programming in sausage visual inspection system
}

\author{
Liang $\mathrm{Gao}^{\dagger}$, Shuai Chen and Yue Ma \\ Shenyang Institute of Automation Chinese Academy of Sciences \\ Shenyang, Liaoning, China \\ ${ }^{\dagger}$ E-mail: gaoliang@sia.cn
}

\begin{abstract}
In order to overcome the influence of black region or complicated texture and extract a smooth contour, this paper provides a novel contour extraction algorithm based on twice dynamic programming. The final contour is extracted by a weighted average of the two contours, and control points optimization method is used to improve real-time and accuracy performance. The algorithm has been used in a sausage visual inspection system, and is proved effective in extracting smooth contour of sausages with complicated texture.

Keywords: Contour Extraction; Dynamic Programming; Visual Inspection.
\end{abstract}

\section{General Instructions}

Machine vision inspection systems have been widely used in many industries to replace the traditional manual inspection. In order to improve the inspection level of sausage, a visual on-line inspection system is designed to inspect the sausage defects according to this paper. It has great significance in protecting the food safety.

Extraction of smooth sausage contour is a key problem in this machine vision system. Early contour extract algorithms mainly focus on enhancing and extracting the high frequency information of images[1]. These methods are efficient, but they can hardly get a complete contour when the background or the foreground isn't segmented well. Algorithms based on level set method have the advantage of stability and topology-independent[2], but they are difficult to obtain good results when extracting discrete edges. Active contour models consider the contour of target region as a continuous and closed chain structure[3], and these models obtain the optimum contour by solving the minimum energy, but the computational complexity is so high that they are not suitable in on-line inspection system. 
For these reasons, a contour extraction algorithm based on dynamic programming is proposed in this paper.

\section{Contour Extraction based on Dynamic Programming}

In this visual inspection system, the region of interest is segmented into three parts. The left and right parts are used to inspect the aluminum buckle and the middle part is used to detect partial raise or depression. The segmentation is shown in Fig.1. To detect partial raise or depression, the contour of middle part is extracted and the diameter value is calculated at each point based on the contour, as shown in Fig.2. Defects can be detected by the deviation of diameter values.

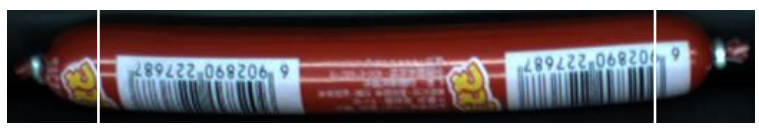

Fig. 1. The segmentation of sausage

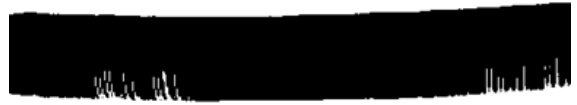

Fig. 2. Contour with mistake

If the sausage surface contains some black regions, there will be a gap on the contour shown in Fig. 2. The gap on the contour may lead to a mistake and the misjudgment rate of the system will rise. In order to overcome the interference of black region or complicated texture, a contour extraction algorithm based on twice dynamic programming is proposed.

\subsection{Contour extraction based on dynamic programming}

The contour extraction based on dynamic programming can be divided into two steps: preparation of the contour data and contour compensation.

Step1:image binary operation is performed and the contour is extracted. As the upper and lower edges are the interesting region, the points of the vertical edges are ignored. the points of each edge are arranged into a row by their $\mathrm{X}$ values. After this arrangement, the edge is present by a sequence of $\mathrm{Y}$ values of the contour points. If there are several points with the same $\mathrm{X}$ value, the outside one will be selected. Through this method, a two-dimensional data is transformed to a one-dimensional data.

Step2: compensation values for each point are calculated. If a point values $Y$ and the compensation value is $d$, the corrected value will be $Y+d$. 
Compensation values are determined by the dynamic programming algorithm. The model can be illustrated according to the following several aspects:

(1)Stage: each compensation value is calculated as a stage. This design meets the requirement of ineffectiveness of dynamic programming.

(2) State: every possible compensation value is adopted as a state.

(3)Target function: target function is designed to evaluate the whole contour. A compensated contour should be continuous, smooth and close to the real contour enough[5]. So the target function is designed as Eq.(1)

$$
E(d)=E_{d}(d)+E_{\text {smooth }}(d)
$$

$E_{d}$ is a data item and $E_{\text {smooth }}$ is a smooth item.

\subsection{Construction of target function}

The essence of the contour extraction algorithm based on dynamic programming is to find the global optimal solution for each point. A perfect result is a contour which is smooth in the barcode region and retains the features of partial raise or depression at the same time.

Data item in the target function is designed to keep the compensated contour points close to the original contour points. A small compensation value can keep the features of partial raise or depression. But in a barcode region, a larger compensation value can make the final contour smooth. In order to satisfy the two conditions at the same time, the data item is designed as Eq.(2)

$$
D(p)=\left\{\begin{array}{cc}
m \times d & Y_{\text {max }}-Y_{\min }<T_{E} \\
S_{d} & \text { other }
\end{array}\right.
$$

In Eq.(2), $Y_{\max }$ and $Y_{\min }$ are the maximum and minimum $\mathrm{Y}$ values of the points around current point. $T_{E}$ is a predefined threshold, $m$ is a coefficient of data item, $d$ is the state of current stage and $S_{d}$ is a predefined constant. In general region, $Y_{\max }-Y_{\min }$ is usually less than $T_{E}$. In this case, the data item is proportional to the compensation value $d$. The contour will be forced to extend along the original contour. In a barcode region, $Y_{\max }-Y_{\min }$ will be large and the original contour points are incorrect. So the data item is not proportional to the value of $d$. The value of $S_{d}$ is usually small to encourage the algorithm to choose a larger $d$ which is large enough to compensate a gap.

Smooth item is proposed to restrict the discontinuity of contour. It is designed based on the difference of $\mathrm{Y}$ values between the current point and its former point. A larger difference means a more discontinue contour and the smooth item will be larger. Similarly, a smaller difference means a smooth 
contour and the punishment should be small too. The smooth item in this paper is proposed as Eq. (3) and Eq. (4)

$$
\begin{gathered}
\mathrm{V}(p)=\left\{\begin{array}{cc}
n \times \Delta d(p) & \Delta d(p)<T s \\
S_{E} & \text { other }
\end{array}\right. \\
\Delta d(p)=Y(p)-Y(p-1)
\end{gathered}
$$

A cut-off value $T_{s}$ is adopted in this paper to avoid serious mistakes. If a point of contour deviates from the real contour a lot, the following points can return to the optimal state with the effect of $S_{E}$.

\section{Optimization of Contour Extraction Algorithm}

The algorithm can overcome the influence of barcode region and achieves a smooth contour, but it still has some deficiency.

First, the compensated contour may deviate from the real contour and tend to form a horizontal line at the end part. It is because there are few following points in the end part, and the effect of smooth item is stronger than data item. Second, the algorithm can hardly achieve the real-time target.

\subsection{Twice dynamic programming}

The dynamic programming is performed twice in different directions. The first one is from left to right and the second one is from right to left.

As inherent defect of dynamic programming, the first contour may be inaccurate at the right side and the second result contour may be inaccurate in left side. In order to solve this problem, the final result can be acquired by taking a weighted average of the two contours. Assuming that the $\mathrm{Y}$ value of the point of first contour is $d_{l}(x)$, and the $\mathrm{Y}$ value of the point of second contour is $d_{r}(x)$, and the number of the points is $L$, then the $\mathrm{Y}$ value of final result can be determined by the Eq.(5), Eq.(6) and Eq.(7).

$$
\begin{gathered}
d(x)=k_{l}(x) \times d_{l}(x)+k_{r}(x) \times d_{r}(x) \\
k_{l}(x)=\frac{L-x}{L} \\
k_{r}(x)=\frac{x}{L}
\end{gathered}
$$




\subsection{Control points}

In this method, some credible points are selected and the contour is forced to pass through these points[6]. It has two advantages.

First, if the selected control points are accurate enough, we will make fewer mistakes. At the same time, control points can guide their neighbor points in the dynamic programming process.

Second, if a contour point is selected as a control point, the computation complexity is reduced.

Control points are selected based on the following principles:

(1)A control point has to be in a smooth part of contour.

(2)The $Y$ value of a control point should be larger than average.

(3)The brightness of some neighboring pixels should be high.

\section{Results of Extraction and Inspection}

The on-line sausage inspection system has been used in industrial field, and all the records and pictures are taken from practical production process.

\subsection{Contour extraction result}

Some pictures of sausages are listed below with the extraction result. In the result image, a white region presents a preliminary extraction result and the contour is the final result.
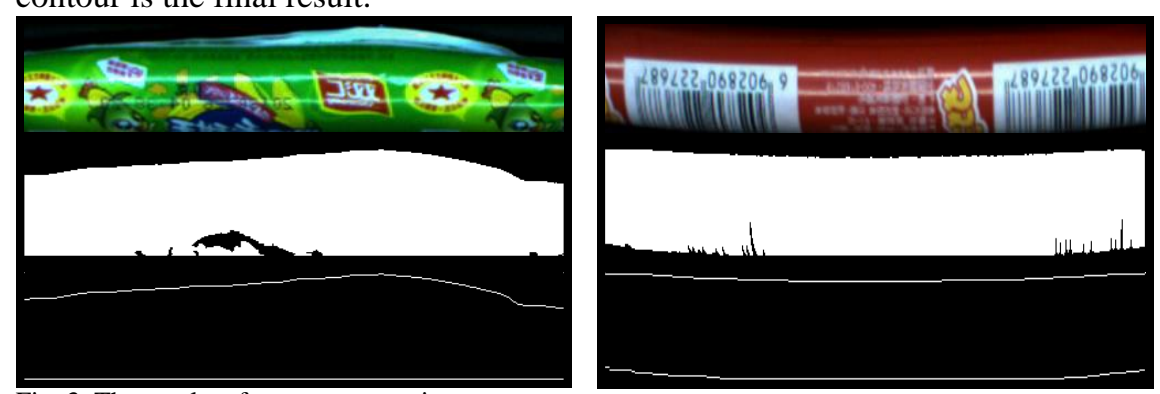

Fig. 3. The results of contour extraction

As shown in the Fig.3, the algorithm proposed in this paper can overcome the influence of barcode region or other dark region, and retain the shape of partial raise. It meets the needs of sausage inspection completely.

\subsection{Precision statistics of the sausages visual inspection}

The precision of this system is measured by counting the number of undetected defectives, and the statistics of the quality rate result is listed in the Table 1. 
Table 1. Statistics of the quality rate

\begin{tabular}{llcc}
\hline style & total & qualified & percent of pass \\
\hline wZw & 10000 & 9997 & $99.97 \%$ \\
xtw & 10000 & 9998 & $99.98 \%$ \\
jkw & 10000 & 9997 & $99.97 \%$ \\
pm & 10000 & 9998 & $99.98 \%$ \\
ptc & 10000 & 9998 & $99.98 \%$ \\
jgw & 10000 & 9998 & $99.98 \%$ \\
jrc & 10000 & 9998 & $99.98 \%$ \\
\hline
\end{tabular}

The statistics shows that the system can achieve a quality rate over $99.95 \%$, it can meet the requirements of quality very well.

\subsection{Real-time performance analysis}

Real-time performance is very important in an on-line inspect system. The time consuming has nothing to do with the sausage styles, but is positive correlate to the size. The time results of inspecting sausages in different sizes range from $30 \mathrm{~g}$ to $60 \mathrm{~g}$ are recorded in Table. 2 .

Table 2. Time of inspecting

\begin{tabular}{cc}
\hline size & average time \\
\hline 30 & $32 \mathrm{~ms}$ \\
40 & $37 \mathrm{~ms}$ \\
50 & $43 \mathrm{~ms}$ \\
60 & $51 \mathrm{~ms}$ \\
\hline
\end{tabular}

Most of the time consuming is less than 50ms. The system can inspect 10 sausages per second easily.

\section{Conclusion}

A contour extraction algorithm is proposed to get an accurate and smooth contour in this paper. A primary extracted contour was compensated in this algorithm. In order to improve the extraction and real-time performance, control point method is adopt to optimize the dynamic programming process. Actual results show that the extraction algorithm proposed in this paper can extract a contour without be affected by barcode region or some other printing factors.

\section{References}

1. Martin D. R., Fowlkes C. C. and Malik. J., Learning to Detect Natural Image Boundaries Using Local Brightness, Color, and Texture Cues, IEEE Trans. On Pattern Analysis and Machine Intelligence, 2004, 26(5): 530549. 
2. Ren X. F., Multi-Scale Improves Boundary Detection in Natural Images. Proceedings of the 10th European Conference on Computer Vision, 2008, 533-545.

3. Shumin Liu, Xujuan Xu, Yingping Huang,. Anti-noise Snake Model and Application in Pedestrian Contour Extraction Information and Control, 2015, 44(5), 564-569.

4. Dreyfus S E, Law A M. The Art and Theory of Dynamic Programming. New York: Academic Press, 1977.

5. Tang Yanfu. Dynamic Programming Stereo Matching Algorithm based on Region Segmentation. Journal of Graphics, 2015, 36 (1): 90-94.

6. Eum H, Kim Y, Palmer R N. Optimal drought management using sampling stochastic dynamic programming with a hedging rule. Journal of Water Resources Planning and Management, 2010, 137(1): 113-122. 